\title{
WHEN IS $C(X)$ A COHERENT RING?
}

\author{
CHARLES W. NEVILLE
}

(Communicated by Louis J. Ratliff, Jr.)

ABSTRACT. We prove: $C(X)$ is coherent if and only if $X$ is basically disconnected.

\section{INTRODUCTION}

In this note, we shall give a topological characterization of those spaces $X$ for which the ring $C(X)$ is coherent. We shall also give a very simple proof of Brookshear and DeMarco's result on when the ring $C(X)$ is semihereditary.

Of course, $C(X)$ denotes the ring of real valued continuous functions on $X$, and $C^{*}(X)$ denotes the ring of bounded functions in $C(X)$. All topological spaces are assumed to be completely regular. A space $X$ is basically disconnected if the closure of every cozero set is again open. The cozero set of a function $f \in C(X)$ is the set $\operatorname{coz}(f)=\{x \in X: f(x) \neq 0\}$. The closed support of $f$ is the set $\operatorname{supp}(f)=\operatorname{clcoz}(f)$. (We shall always denote the closure of a set $A$ by $\operatorname{cl} A$ ). Thus $X$ is basically disconnected if and only if the closed support of every function $f \in C(X)$ is open.

Basically disconnected spaces often seem very unreasonable, so it is important to realize that they occur in a very natural algebraic context: A compact space $X$ is basically disconnected if and only if it is homeomorphic to the Stone space (space of maximal ideals) of a Boolean $\sigma$-algebra [S, 22.4].

All rings are assumed to be commutative with a unit element. For unexplained algebraic terminology, the reader should consult either of the books by Rotman $\left[R_{1}, R_{2}\right]$. (Another excellent reference will be the book by Glaz [Gz], when it appears.) For unexplained topological terminology, the reader should consult the book by Gillman and Jerison [GJ].

Received by the editors April 11, 1988 and, in revised form, June 9, 1988.

1980 Mathematics Subject Classification (1985 Revision). Primary 54C40; Secondary 13C10, 13C11, 13C12, 13D05, 13F05, 13E15, 54G05, 54G10.

Key words and phrases. Projective module, free module, coherent ring, semihereditary ring, basically disconnected space, Hausdorff space, completely regular space, topological space, continuous function, ring of continuous functions.

This work was done while the author was on leave at Wesleyan University. 


\section{Brookshear AND DeMarco's theorem}

The following beautiful theorem is due to Brookshear $\left[\mathrm{BR}_{2}\right]$ and DeMarco [DM]:

Theorem 1.1. $C(X)$ is semihereditary; that is, every finitely generated ideal of $C(X)$ is projective if and only if $X$ is basically disconnected.

A simple proof can be based on the following standard lemma $\left[R_{1}\right.$, Lemma 4.15].

Lemma 1.2. Let $R$ be a ring. An $R$ module $A$ is projective if and only if there are elements $\left\{a_{k}: k \in K\right\}$ of $A$ and homomorphisms $\varphi_{k}: A \rightarrow R$ such that

(1.2.1) if $a \in A, \varphi_{k}(a)=0$ for all but finitely many $k$;

(1.2.2) if $a \in A, a=\sum_{k}\left(\varphi_{k}(a)\right) a_{k}$.

It follows from the proof in $\left[\mathrm{R}_{1}\right]$ that if $A$ has a generating set $G$ then $\left\{a_{k}: k \in K\right\}$ may be chosen to equal $G$. The set $\left\{a_{k}: k \in K\right\}$ together with the homomorphisms $\varphi_{k}$ is often called a projective basis for $A$.

Proof of Theorem 1.1. Suppose $C(X)$ is semihereditary. Let $f \in C(X)$ and consider the principal ideal $I=(f) . I$ is projective, so there exists a projective basis $a_{1}, \varphi_{1}$ with $a_{1}=f$. For any $g \in C(X)$, we have the pair of equations

$$
\begin{aligned}
g f & =\varphi_{1}(g f) f \\
\varphi_{1}(g f) & =g \varphi_{1}(f)
\end{aligned}
$$

the second equation being true because $\varphi_{1}$ is a $C(X)$ module homomorphism. We can cancel the right-hand $f$ 's in the first equation wherever $f \neq 0$. Thus $\varphi_{1}(f)=1$ on the closed support of $f, \operatorname{supp}(f)$. (Remember, $\varphi_{1}(f) \in C(X)$ since $\varphi_{1}: I \rightarrow C(X)$.) Suppose, to reach a contradiction, that $\operatorname{supp}(f)$ is not open. We shall show that $\varphi_{1}$ is not well defined. Since $\operatorname{supp}(f)$ is not open, there exists $x \in X \sim \operatorname{supp}(f)$ such that $\varphi_{1}(f)(x) \neq 0$, and there exist $g_{1}$ and $g_{2} \in C(x)$ such that $g_{1}=g_{2}$ on $\operatorname{supp}(f)$, but $g_{1}(x) \neq 0$ and $g_{2}(x)=0$. Then $g_{1} f=g_{2} f$, so $\varphi_{1}\left(g_{1} f\right)=\varphi_{1}\left(g_{2} f\right)$. However, $\varphi_{1}\left(g_{1} f\right)(x) \neq \varphi_{1}\left(g_{2} f\right)(x)$, since the second of the equations (1.3) shows $\varphi_{1}\left(g_{1} f\right)(x) \neq 0$ and $\varphi_{1}\left(g_{2} f\right)(x)=0$. This is a contradiction, so $\operatorname{supp}(f)$ is open. Thus the closure of every cozero set is open, so $X$ is basically disconnected.

Conversely, suppose $X$ is basically disconnected. Let $I$ be a finitely generated ideal. Now $X$ is an $F$ space, so every finitely generated ideal is principal [GH; GJ, Theorem 14.25]. Thus $I=(f)$. Because $X$ is basically disconnected, $K=\operatorname{supp}(f)$ is clopen, so $\chi_{k}$, the characteristic function of $K$, is continuous. Let $\varphi_{1}(g f)=g \chi_{k}$, for all $g \in C(X)$. Then $\left(a_{1}, \varphi_{1}\right)=\left(f, \varphi_{1}\right)$ is a projective basis for $I$, so $I$ is projective.

From this proof, we have

Corollary 1.4. $X$ is basically disconnected if and only if every principal ideal of $C(X)$ is projective. 
Brookshear and DeMarco also proved that $C(X)$ is hereditary, that is every ideal of $C(X)$ is projective, if and only if $X$ if finite and discrete $\left[\mathrm{BR}_{2}\right.$, DM]. Swan has characterized all finitely generated projective $C(X)$ modules for compact Hausdorff $X$ : A finitely generated $C(X)$ module is projective if and only if it is isomorphic to the module $\Gamma(\xi)$ of all sections of $\xi$ over $X$, for some vector bundle $\xi$ over $X[\mathrm{Sw}]$.

\section{WhEN IS $C(X)$ A COHERENT RING?}

For rings, the next best thing to being semihereditary is to be coherent. A ring $R$ is coherent if every finitely generated ideal is finitely presented. This means that there exists an exact sequence

$$
0 \rightarrow K \rightarrow F \rightarrow I \rightarrow 0,
$$

where $F$ is a finitely generated free $R$ module and $K$ is also finitely generated.

Theorem 2.2. $C(X)$ is coherent if and only if $X$ is basically disconnected.

Proof. Suppose $C(X)$ is coherent. As in the proof of Theorem 1.1. we consider a principal ideal $I=(f)$. We have the exact sequence

$$
0 \rightarrow K \rightarrow C(X) \stackrel{\varphi}{\rightarrow} I \rightarrow 0
$$

where $\varphi(g)=f g . I$ is finitely presented. Now the exact sequence (2.3) may be different from the exact sequence (2.1) with a finitely generated kernel, but it is an easy corollary of Schanuel's lemma [ $\mathrm{R}_{1}$, Corollary 3.42] that $K$ in (2.3) is finitely generated. $K$ is particularly easy to describe:

$$
K=\{k \in C(X): \operatorname{coz}(k) \cap \operatorname{supp}(f)=\varnothing\}
$$

where $\cos (k)$ is the cozero set of $k$. Let $k_{1}, \ldots, k_{n}$ generate $K$. Consider $h=\left|k_{1}\right|+\cdots+\left|k_{n}\right|$. Since $\operatorname{coz}(h) \cap \operatorname{supp}(f)=\varnothing, h \in K$ as does $\sqrt{h}$. We claim

$$
K=\{k \in C(X):|k| \leq g h \text { for some } g \in C(X)\}
$$

First, if $|k| \leq g h$ then $\operatorname{coz}(k) \subseteq \operatorname{coz}(h)$, so $k$ satisfies (2.4) and thus belongs to $K$. Conversely, if $k \in K$, then $k=g_{1} k_{1}+\cdots+g_{n} k_{n}$ with $g_{1}, \ldots, g_{n} \in C(X)$, so $|k| \leq\left(\Sigma|g|_{i}\right) h$. Thus the claim is true. One simple consequence of (2.5) is that $\operatorname{coz}(h)=\bigcup\{\operatorname{coz}(k): k \in K\}=X \sim \operatorname{supp}(f)$. Another is that since $\sqrt{h} \in K, \sqrt{h} \leq g h$ for some $g \in C(X)$. In other words, there exists $g \in C(X)$ such that

$$
1 \leq g \sqrt{h}
$$

on $\operatorname{coz}(h)$. We claim this shows $\operatorname{coz}(h)$ is closed. Let $p \in \operatorname{cl} \operatorname{coz}(h)$. Let $\left(x_{\alpha}\right)$ be a net in $\operatorname{coz}(h)$ converging to $p$. By (2.6) and the continuity of $g$,

$$
1 \leq \lim _{\alpha} g\left(x_{\alpha}\right) \sqrt{h\left(x_{\alpha}\right)}=g(p) \sqrt{h(p)}
$$


so $h(p)>0$. Thus $p \in \operatorname{coz}(h)$. This establishes the claim. Since $\operatorname{coz}(h)$ is closed, $\operatorname{supp}(f)$ is open. Hence the closure of every cozero set is open, so $X$ is basically disconnected.

Conversely, suppose $X$ is basically disconnected. Let $I$ be a finitely generated ideal. As in the proof of Theorem 1.1, X is an $F$ space so $I=(f)$. Again we have the exact sequence $(2.3)$. Since $X$ is basically disconnected, $\operatorname{supp}(f)$ is clopen. The characteristic function of $\operatorname{supp}(f), \chi_{\operatorname{supp}(f)}$, generates $K$. Thus $K$ is finitely generated, so $I$ is finitely presented.

Every semihereditary ring is coherent, since if $I$ is a finitely generated projective ideal the exact sequence (2.1) splits, and thus $K$ is also finitely generated. Hence we can deduce the lengthy part of Theorem 1.1 , that $C(X)$ semihereditary implies $X$ basically disconnected, from Theorem 2.2.

Theorem 2.2 is surprising for the following reason: As we have just seen, coherence is formally weaker than the property of being semihereditary. Usually, if one considers a class of rings, there are many coherent rings in the class which are not semihereditary. But for rings of type $C(X)$, a ring is coherent if and only if it is semihereditary.

Note added in proof. It has come to the attention of the author that one of the theorems in this paper, $C(X)$ is coherent if and only if $X$ is basically disconnected, was discovered for compact spaces by Lawrence G. Brown and Heinrich Lotz in 1976. The theorem was quoted without proof by W. S. McVoy and L. A. Rubel in their paper, Coherence of some rings of functions, J. Funct. Anal. 21 (1976), p. 86, where they referenced a private communication from Brown and Lotz. Lawrence G. Brown has written me that no proof ever appeared in print.

\section{REFERENCES}

$\left[\mathrm{BR}_{1}\right]$ J. G. Brookshear, Projective ideals in rings of continuous functions, Pacific J. Math. 71 (1977), 313-333.

[BR $\left.\mathrm{BR}_{2}\right]$, On projective prime ideals in $C(X)$, Proc. Amer. Math. Soc. 69 (1978), 203-204.

[DM] G. DeMarco, Projectivity of pure ideals, Rend. Sem. Mat. Univ. Padova 68 (1983), 289-304.

[GH] L. Gillman and H. Henriksen, Rings of continuous functions in which every finitely generated ideal is principal, Trans. Amer. Math. Soc. 82 (1956), 366-391.

[GJ] L. Gillman and M. Jerison, Rings of continuous functions, Van Nostrand, New York, 1960.

[Gz] S. Glaz, Commutative coherent rings, Lecture Notes in Math., vol. 1371, Springer-Verlag, New York, 1989.

$\left[\mathrm{R}_{1}\right] \quad J$. Rotman, Notes on homological algebra, Van Nostrand, New York, 1970.

$\left[\mathrm{R}_{2}\right]$, An introduction to homological algebra, Academic Press, New York, 1979.

[S] R. Sikorski, Boolean algebras, 3rd ed., Springer-Verlag, New York, 1969.

[Sw] R. Swan, Vector bundles and projective modules, Trans. Amer. Math. Soc. 105 (1962), 264-277.

Department of Mathematics, Wesleyan University, Middleton, Connecticut, 06457

Current address: Department of Computer Science, Central Connecticut State University, New Britain, Connecticut 06050 